\title{
CONOCIMIENTOS Y PERCEPCIONES VINCULADAS CON EL VIH/ SIDA EN COMUNIDADES SHUAR DEL ECUADOR
}

\author{
KNOWLEDGE AND PERCEPTIONS LINKED TO HIV/AIDS IN SHUAR \\ COMMUNITIES OF ECUADOR
}

\section{CONHECIMENTO E PERCEPÇÓES RELACIONADAS AO HIV/AIDS NAS COMUNIDADES SHUAR DO EQUADOR}

\author{
Liliana Alexandra Brito Roby* \\ Doriz ANGÉLICA JimÉNEZ BRITO** \\ Elisabeth Cecilia Sinche Rivera*** \\ Aydée Narcisa Angulo Rosero****
}

\begin{abstract}
RESUMEN
Objetivo: Analizar los conocimientos y las percepciones vinculadas con el VIH/SIDA en comunidades Shuar de la parroquia Sevilla Don Bosco - Provincia Morona Santiago, Ecuador. Material y Método: Estudio cualitativo fenomenológico, cuyas técnicas de investigación fueron entrevistas en profundidad y grupos focales. La muestra fue seleccionada por conveniencia; participaron 40 personas entre hombres y mujeres de 14 a 50 ańos. Los criterios de inclusión fueron: personas Shuar con vida sexual activa y no diagnosticadas con VIH/SIDA. Las categorías de análisis fueron la información, conocimientos y percepciones respecto al VIH/SIDA. Resultados: Los datos reflejan que en las comunidades de Sevilla Don Bosco existe un acceso a la información condicionado por la interacción de la comunidad con las instituciones de salud pública, instituciones educativas y tecnologías de la información y comunicación; los conocimientos y las percepciones sobre el VIH/SIDA varían por género, edad, nivel de educación y el lugar de vivienda de los participantes. Conclusiones: En la comunidad Shuar se expresan limitaciones e imprecisiones de los conceptos y procesos propios del virus y la enfermedad; el tema circula como rumor; los adolescentes mayormente obtienen información en los colegios y por medio de la internet; conocen mejor el VIH/SIDA los hombres y los adolescentes en general; las mujeres tienen limitaciones para expresar conocimientos y opiniones; la enfermedad ha sido estigmatizada; la vulnerabilidad de la población frente a la inmunodeficiencia y el peligro de las enfermedades oportunistas es clara en la comunidad; manifiestan interés y disposición a conocer mejor el VIH/SIDA.
\end{abstract}

Palabras clave: VIH/SIDA; Conocimiento, Percepción, Etnia.

\footnotetext{
"Socióloga, Investigadora de la Dirección de Investigación de la Universidad de Cuenca. Cuenca-Ecuador, Email: liliana.britor@ucuenca.edu.ec, Autor de correspondencia.

"Licenciada en Enfermería, Docente Investigadora de la Facultad de Ciencias Médicas de la Universidad de Cuenca. CuencaEcuador. Email: doriz.jimenez@ucuenca.edu.ec

"'Licenciada en Enfermería, Docente Investigadora de la Facultad de Ciencias Médicas de la Universidad de Cuenca. CuencaEcuador. Email: elisabeth.sinche@ucuenca.edu.ec

"*."Licenciada en Enfermería, Docente Investigadora de la Facultad de Ciencias Médicas de la Universidad de Cuenca. CuencaEcuador. Email: aydee.angulo@ucuenca.edu.ec
} 


\begin{abstract}
Objective: To analyze the knowledge and perceptions related to HIV/AIDS in Shuar communities from the parish of Sevilla Don Bosco, Morona-Santiago Province, Ecuador. Materials and Methods: Qualitative phenomenological study using following research techniques: in-depth interviews and focus groups. A convenience sample was used: 40 men and women aged 14 to 50 years participated. The inclusion criteria corresponded to Shuar people with active sexual life and without HIV/AIDS diagnosis. The categories of analysis were information, knowledge, and perceptions regarding HIV/AIDS. Results: The data show that in the communities of Sevilla Don Bosco there is access to information conditioned by the interaction of the community with public health institutions, educational institutions, and information and communication technologies. Knowledge and perceptions regarding HIV/AIDS vary according to gender, age, level of education, and place of residence of the participants. Conclusions: In the Shuar community, limitations and inaccuracies about the concepts and processes of the virus and the disease are expressed. The subject circulates as a rumor. Adolescents obtain information mostly in schools and through the Internet. In general, men and young people have more knowledge about HIV/AIDS. Women have limitations to express knowledge and opinions. The disease has been stigmatized. The population's vulnerability against immunodeficiency and the danger of opportunistic diseases is clear in the community. They show interest and willingness to learn more about HIV/AIDS.
\end{abstract}

Key words: HIV/AIDS; Knowledge; Perception; Ethnicity.

\title{
RESUMO
}

Objetivo: Analisar os conhecimentos e as percepçóes relacionadas ao HIV/AIDS nas comunidades Shuar da paróquia Sevilla Don Bosco - Morona Santiago, Equador. Material e Método: Estudo qualitativo fenomenológico, as técnicas de investigação foram entrevistas em profundidade e grupos focais. A amostra foi selecionada por conveniência; participaram 40 pessoas entre homens e mulheres de 14 a 50 anos. Os critérios de inclusão foram: pessoas Shuar com vida sexual ativa e sem diagnóstico de HIV/AIDS. As categorias de análise foram a informação, os conhecimentos e as percepçóes em relação ao HIV/AIDS. Resultados: Os dados concluíram que nas comunidades de Sevilla Don Bosco existe acesso à informação condicionado pela interação da comunidade com as instituiçóes de saúde, instituiçôes de educação e tecnologias da informação e comunicação; os conhecimentos e as percepçôes sobre o HIV/AIDS são diferentes segundo o gênero, a idade, o nível de educação e o local de residência dos participantes. Conclusóes: Na comunidade Shuar ficam expressas limitaçóes e imprecisões dos conceitos e dos processos próprios do vírus e da enfermidade; o assunto circula como rumor; os adolescentes com frequência obtêm informação das escolas e através da internet; em geral, homens e adolescentes conhecem mais o HIV/AIDS; as mulheres possuem limitaçóes para expressar conhecimentos e opinióes; a enfermidade tem sido estigmatizada; a vulnerabilidade da população frente à imunodeficiência e o perigo das doenças oportunistas são claros na comunidade; manifesta-se interesse e disposição para conhecer melhor o HIV/AIDS.

Palavras-chave: HIV/AIDS; Conhecimento, Percepção, Etnia.

Fecha de Recepción: 13/11/2018

Fecha de Aceptación: 07/05/2019

\section{INTRODUCCIÓN}

En el año 2016 la Organización de las Naciones Unidas para el $\mathrm{SIDA}^{(1)}$ informó que existen alrededor de 36,7 millones de personas que viven con VIH y el 53\% de los reportados recibían terapia antirretroviral. A mediados de 2017, alrededor de 20,9 millones de personas recibían esa terapia en todo el mundo ${ }^{(2-4)}$. Varias investigaciones han demostrado que la posibilidad de infección por el VIH está en crecimiento por las conductas riesgosas, tanto en hombres como mujeres, sin importar la edad, las preferencias u orientaciones sexuales.

La aparición de casos de VIH en comunidades 
indígenasdeAméricadatade 1996, cuyainformación proviene especialmente de Canadá y los Estados Unidos. Del resto del continente la información es muy incierta debido principalmente a que los reportes de nuevos casos no consideran la variable étnica ya que la categoría indígena supone mayor complejidad $^{(5,6,7)}$. Aun así, se conoce la prevalencia de comunidades indígenas de Honduras, Panamá, Suriname y Canadá, que es seis veces más alta que en la población en general, condicionada por los procesos migratorios, la exclusión social y cultural y el uso, cada vez mayor, de drogas ${ }^{(8)}$.

En vista de que el número de integrantes de comunidades étnicas que viven con VIH/SIDA se ha incrementado, algunos países como Colombia han propuesto plantear una alerta para que las investigaciones en el área de las ciencias humanas y de la salud asuman la epidemia como un fenómeno social particularizado ${ }^{(9,10)}$.

El Ministerio de Salud Pública de Ecuador ${ }^{(11)}$, en el año 2016, reportó 4.862 casos de VIH, de los cuales 3.183 fueron hombres y 1.679 mujeres. De acuerdo a ONUSIDA ${ }^{(12)}$, la estimación de adultos y nińos viviendo con VIH/SIDA en Ecuador al 2017 es de 42.000 [28.000 - 69.000].

De acuerdo a Alderete ${ }^{(13)}$, los índices de mortalidad y morbilidad reflejan inequidades y obstáculos en el acceso al derecho a la salud por las comunidades indígenas, así como limitaciones para generar y administrar la información de salud. Señala que la situación sanitaria de los pueblos nativos está determinada, en gran medida, por su bajo nivel socioeconómico, por los cambios de los modos de vida tradicional y los patrones alimentarios y conductuales.

Varios autores concuerdan que las investigaciones relacionadas con las percepciones sobre el VIH/SIDA deben aproximarse al contexto sociocultural y de género para poder intervenir adecuadamente en los marcos de cada cultura ${ }^{(3,14)}$. Investigaciones de los últimos ańos realizadas en comunidades indígenas de América Latina reflejan un conocimiento limitado sobre varios aspectos de la enfermedad: conceptos de VIH/SIDA, signos y síntomas, formas de transmisión, diagnóstico y prevención, e incluso, algunos autores han mencionado que las comunidades consideran el SIDA como una enfermedad nueva, cuya implicación cultural sería la ruptura del sistema de clasificación tradicional de las enfermedades ${ }^{(7,15,16)}$.
En este contexto, el conocimiento inadecuado y distorsionado referido a la salud sexual puede favorecer a que se mantengan costumbres, tabúes, mitos o tradiciones de origen étnico o religioso que, traducidas en conductas riesgosas, les sigue la consecuente proliferación del virus ${ }^{(17)}$.

En virtud de lo señalado, el objetivo del presente estudio fue analizar los conocimientos y las percepciones vinculadas con el VIH/SIDA, en comunidades Shuar de la parroquia Sevilla Don Bosco, ubicada en el cantón Morona, provincia de Morona Santiago del Ecuador. Se eligió esta comunidad por ser la más extensa, con 97 asentamientos humanos clasificados en 12 barrios y 85 comunidades, cuya población, en su mayoría (> $80 \%)$, está compuesta por mujeres y hombres que se autoidentifican como indígenas ${ }^{(18)}$.

$\mathrm{Al}$ acercarse, indagar y comprender esta realidad social, desde la subjetividad y bajo un enfoque cualitativo, no solo se puede rescatar valiosa información para retroalimentar las intervenciones en salud, también se fortalece la investigación local e internacional de las categorías VIH/SIDA y etnia.

\section{MATERIAL Y MÉTODO}

Tipo de estudio: fue cualitativo fenomenológico, interpretativo, ya que permite una aproximación a la realidad (significados y sentidos) sobre los conocimientos y percepciones relacionados con el fenómeno de estudio, el problema y objetivos de la investigación.

Muestra: se seleccionó por conveniencia y se obtuvo con la colaboración de autoridades de los centros de salud del Ministerio de Salud Pública del Ecuador (MSP), que junto con el equipo de investigación, de forma previa, socializaron la propuesta con la comunidad y los líderes comunitarios. No hubo resistencia hacia la participación de ningún miembro de la comunidad, al contrario, hubo interés y conciencia de la necesidad del estudio. Los criterios de inclusión fueron: hombres y mujeres Shuar, con vida sexual activa, que acudieron a los centros de salud de la comunidad y que firmaron el consentimiento y asentimiento informado. Los criterios de exclusión fueron: habitantes diagnosticados de VIH/SIDA y usuarios de los servicios de salud que tuvieron 
algún tipo de dificultad para la comunicación oral. Debido a la naturaleza del fenómeno de estudio, a la accesibilidad de la población y a los resguardos metodológicos ${ }^{(19)}$, la muestra se constituyó por 40 personas.

Recolección de datos: Se realizaron 4 grupos focales y 12 entrevistas en profundidad, con representación equitativa por edad y género, a saber: 14 adolescentes y 14 adultos en los grupos focales; 6 adolescentes y 6 adultos en las entrevistas a profundidad. Las entrevistas fueron semiestructuradas, tuvieron una duración media de 30 minutos, se realizaron en los hogares de los participantes en diferentes horarios. Los grupos focales se realizaron, para el caso de los adultos, en las casas comunales y para los adolescentes, previo a la aprobación de las autoridades, en horarios de clases de las instituciones educativas. Se consideraron grupos homogéneos representativos (en sentido de prototipo) de los segmentos adultos y adolescentes-estudiantes y en su desarrollo tuvieron una duración media de 50 minutos. Ambas técnicas usaron el mismo temario a fin de triangular los resultados y posteriormente contrastarlos con la literatura. Los grupos focales y las entrevistas se realizaron en español pues no hubo necesidad de un traductor, con el resguardo de que ante cualquier contingencia, los informantes eran bilingües del idioma español y shuar. La información fue grabada previa autorización de los participantes. Los datos fueron recolectados entre mayo y junio de 2017, en las localidades específicas de: Sagrado Corazón, San Luis de Ininkis, Sevilla Don Bosco, Guadalupe y Uyuntza, todas pertenecientes al área de influencia geográfica de Sevilla Don Bosco.

Análisis: Las grabaciones y notas de campo fueron transcritas en formato Word, convirtiéndose en documentos primarios completos. El equipo de investigación multidisciplinario llevó a cabo el análisis del discurso fenomenológico interpretativo, en el cual, el investigador interpreta los significados y sentidos de los participantes en base a las siguientes categorías a priori: a) conocimientos y percepciones; b) categorías emergentes como: edad, estado civil, nivel de instrucción, identidad, ocupación e información. Las interpretaciones fueron discutidas por todos los investigadores. A pesar de que participaron 40 personas en la investigación, para el análisis se consideraron los discursos relevantes al objetivo de investigación, ya sea por las veces que se repetían o porque eran novedosos o inusuales. La herramienta usada para el análisis fue el software Atlas ti, versión 7.5.4.

Para proteger el anonimato y asegurar la confidencialidad de los participantes en la investigación, se utilizaron códigos diseñados acorde a las características de la población: sexo, ciclo vital, ubicación y tipo de técnica. Así, los nombres observados en los resultados se identifican de la siguiente manera: " $M$ " de mujer, " $H$ " de hombre, seguido por el número que se le otorgó al participante 1 al 20: la "A" (mayúscula) de adulto y la "a" (minúscula) de adolescente, seguido por "SB" de Sevilla Don Bosco, "SC" de Sagrado Corazón, "G" de Guadalupe, "SL" de San Luis de Inimkis y "U” de Uyuntza; se incluyeron además las letras "e" y "gf" que corresponden a entrevistas y a grupos focales, respectivamente.

\section{RESULTADOS}

Características sociodemográficas de los participantes: La edad de los adolescentes osciló entre los 14 a 17 ańos y la de los adultos entre los 18 a 50 años. De los adolescentes, el 39\% fueron mujeres y el 61\% hombres; de los adultos, el 53\% fueron mujeres y el $47 \%$ hombres, Todos los participantes se identificaron con la etnia Shuar. Los adolescentes, en su mayoría (95\%), manifestaron ser solteros, salvo uno que señaló mantener una relación de unión libre; de los adultos, la mayoría (65\%) mantenía relaciones de unión libre. En cuanto al nivel de instrucción, el 23\% de los participantes indicaron haber cursado la primaria y el $77 \%$ el bachillerato. La mayoría de los adultos (60\%) se dedican a la caza y al campo, mientras que la mayoría de los adolescentes se dedican al estudio (90\%) y al trabajo (10\%).

Información del VIH/SIDA: La información que tienen los participantes respecto al VIH/SIDA es limitada:

Sí, hemos escuchado sobre VIH/SIDA, pero no sabemos bien las características de cómo se puede transmitir esa enfermedad, no sabemos tanto. H18.A.U.gf.

La mayor información proviene de la escuela y de los medios de comunicación tradicionales; además, 
la que se recibe en los centros de salud, de parte de los Técnicos de Atención Primaria de Salud (TAPS), en las ocasionales visitas del personal de salud a las comunidades (charlas, material impreso, videos) y la que se recibe en medio de la atención médica:

A veces acudimos, vamos al centro de salud, a veces el doctor hace charlas alli. H17.A.U.gf.

Señoritas o empleados o enfermero solamente vienen a explicarte. H9.A.SL.e.

La información proveniente de la televisión es menos común, pero importante:

En la televisión, ahi hablan bastante de eso y yo se ver. M8.A.G.gf.

En la televisión, en las noticias (...) escuché eso. M17.A.U.gf.

También hay información en otros espacios no tan comunes:

En los talleres que tuvimos en el trabajo que yo trabajaba entonces ahi nos explicaron más sobre el VIH. M12.A.SL.e.

En el caso de los adolescentes la información se obtiene mayormente en los colegios, es impartida por los docentes y personal especializado del Ministerio de Salud Pública (MSP) como parte de la Estrategia Nacional de Salud Pública para VIH/SIDA:

Nos vinieron a dar una charla, del Subcentro. H14.a.SL.gf.

La información circula como rumor dentro de la familia, con los amigos y en la comunidad:

Se diagnostica, dicen que la persona que tiene VIHSIDA tiene algunos sintomas, dicen que se hace flaco, a veces se engorda, dicen que le da mucha diarrea, no les da esas ganas de comer, eso dicen. H11.A.SL.e.

Los que más dicen que tienen son los viejos porque las mujeres de la calle andan por aquí, por allá y los viejos consumen eso (...) dicen que ellos tienen. M9.A.G.gf.

Los investigados, en su mayoría no buscan información y cuando lo hacen, son mayormente los adolescentes que usan la Internet:

Lo que yo he escuchado por las noticias, por el internet, de investigación ahi que uno se va recopilando asi. M14.A.SL.gf.
Respecto a la demanda de mayor información, se reflejó esta necesidad principalmente en la población adulta y de las comunidades de Uyuntza y de San Luis de Inimkis:

Nosotros tenemos esos conocimientos, pero no tenemos tanto a profundo como es ese VIH/SIDA por eso estoy muy interesado para entender sobre este tema, por tal razón estamos aquí. H18.A.U.gf.

Quiero conocer eso, ¿cómo es ese?, ¿de qué forma es eso?, ¿cómo? o sea conocer y saber. H9.A.SL.e.

Conocimientos del VIH/SIDA: En el grupo de estudio se evidenció que los hombres conocen al VIH/SIDA mejor que las mujeres y los adolescentes más que los adultos.

Las mujeres se limitan en el momento de expresar sus opiniones:

El SIDA exactamente no, no sé, porque dicen que el VIH, no sé si es muy diferente que el SIDA. M10.A.SL.e.

Existe duda y confusión entre VIH y SIDA, mayormente por parte de la población adulta:

¿VIH es igual a SIDA? M7.A.G.gf. Dicen que el VIH, no sé, si es muy diferente que el SIDA. H10.A.SL.e.

En algunos casos, ambos términos fueron identificados como enfermedad:

EL VIH es una enfermedad. H13.a.SL.gf.

Unos pocos informantes respondieron de forma precisa:

El VIH es solamente el virus que luego se va formando y se produce el SIDA. M4.a.SC.e.

Un virus inmune, algo así. M10.A.SL.e.

Síndrome de Inmunodeficiencia Adquirida. M14.A.SL.gf.

Pocos informantes en las diferentes comunidades respondieron acertadamente sobre la transmisión, la mayoría mencionaron que es un asunto de la ausencia de cuidado y acusaron exclusivamente a las relaciones sexuales sin protección, unos pocos mencionaron por vía sanguínea o de madre a hijo durante el embarazo:

Se forma a través de las relaciones sexuales, sin 
preservativos, hacer relaciones asi por hacer no más, sin cuidado. M9.A.G.gf.

La mayoría adquiere por contacto sexual, de eso se adquiere más el VIH. M1.a.SB.gf.

Es un virus que puede ser contagiado mediante las relaciones sexuales o por vía

sanguinea, o de madre a hijo. M4.A.SC.e.

Cuando usan jeringas. H6.a.SC.e.

Persisten algunos conceptos erróneos sobre la transmisión de la enfermedad como: contagiarse por estrechar la mano con un contagiado, compartir un asiento o una cuchara, practicar la masturbación o la falta de aseo:

A través de dar la mano tal vez. H10.A.SL.e.

No ocupar asi la cuchara del que está infectado, porque algunas veces ahi se queda alguna secreción de la sangre y viene a producir esa enfermedad. H11.A.SL.e.

Que causa por el asiento, esa enfermedad, también con masturbación, nos han dicho. H8.A.G.gf.

Hacen relaciones con manos sucias, los hombres no se asean, nos tocan con manos sucias. M8.A.G.gf.

El conocimiento sobre el curso de la enfermedad es adecuado cuando se afirma el largo tiempo que se requiere para que el SIDA aparezca; sin embargo, ninguno de los participantes cita explícitamente el estado de portador:

Están normales, o sea, se ven como si no estuvieran enfermos... dura varios años para aparecer los primeros sintomas. M4.A.SC.e.

Eso no duele, ni pica, eso es poco a poco y lo acaba esa enfermedad. H19.A.U.gf.

El conocimiento, aunque no completo, sobre los signos y síntomas es pertinente; entre los síntomas mencionados encontramos: baja de defensas, variaciones en el peso, diarrea, pérdida de apetito, manchas en la piel, entre otros:

Se hace flaco, a veces se engorda, dicen que les da mucha diarrea, no les da esas ganas de comer, eso dicen. H11.A.SL.e.

En la piel se asoma unas manchas, unas manchas blancas y se vuelve flaco. H10.A.SL.e.

Dicen que cuando ya está avanzando, el cuerpo se hace pequeño, se hace flaco, se queda en coma y ya no comienza a... ¿cómo le digo? su fuerza, se le va todo. M3.A.SB.gf.
Los informantes conocen que el SIDA destruye las defensas del organismo, señalan la vulnerabilidad del cuerpo de contraer otras enfermedades que lo invaden y se constituyen en peligrosas porque pueden provocar la muerte:

Viene acompañado con otras enfermedades que son como la gripe, diarrea, dolor de cabeza entre otros malestares. M1.a.SB.gf.

Están acabadas las defensas, hasta una gripe lo puede matar, eso he escuchado del VIH. M12.A.SL.e.

Percepciones del VIH/SIDA: Cuando los informantes reconocieron al SIDA como una enfermedad, se expresa una percepción negativa y de serio peligro pues la caracterizan como terrible, incurable y mortal:

No tenemos conocimientos, hemos escuchado esa terrible enfermedad. M15.A.U.gf.

El SIDA es una enfermedad mortal, dicen eso. M12.A.SL.e.

Se percibe la capacidad de contagiarse fácilmente y que la enfermedad avanza. También se desconocen los pasos a seguir en caso de que alguien sufriera la enfermedad:

Es algo que pasa (...) el SIDA transmite (...) sigue pasando, pasando por aqui, sigue aumentando. H7.A.G.gf.

Si supiera, conociera esa enfermedad yo te avisaria, pero de todos modos no conozco donde hacer, en que forma, en qué manera puede afectarse o como será en sexo, no sé. H9.A.SL.e.

Uno de los signos mencionados por un participante que llamó la atención es el deseo sexual exacerbado del contagiado, que al parecer al igual que los conocimientos, circula en la comunidad a manera de rumores o comentarios:

Se vuelve desesperada, anda por aqui y por allá, se desespera, según el comentario que yo he oído (...) por el deseo sexual. M7.A.G.gf.

\section{DISCUSIÓN}

Las fuentes de información de los participantes en la investigación variaron según el acceso a instituciones educativas, la influencia de los 
servicios de salud local, los medios de comunicación y las visitas de profesionales o técnicos de atención primaria de salud (TAPS) en la comunidad o en los colegios. Esto concuerda con otros estudios como el de Zambrano et al. ${ }^{(5)}$ sobre conocimientos de VIH y comportamientos sexuales en una comunidad indígena de Antioquía. Cabe hacer notar que no es frecuente el uso de la comunicación radial como fuente de información como sí ocurrió con la población indígena de las tierras bajas de Bolivia en el estudio de Puig y Montalvo ${ }^{(20)}$, sobre conocimientos actitudes y prácticas de ITS y VIH/ SIDA.

La información sobre VIH/SIDA que circula entre la comunidad proviene mayormente de un proceso de socialización informal basada en el rumor, lo que puede distorsionar o causar confusión. Similar situación se observó en un estudio sobre intimidad del buen vivir realizado en Ecuador ${ }^{(21)}$, en donde se calificó la información sobre el tema como fragmentada e imprecisa. Ante esta situación, al igual que otros estudios realizados en comunidades indígenas de Bolivia ${ }^{(20)}$ y Colombia ${ }^{(9)}$, los informantes de esta investigación manifestaron la necesidad de información de mejor calidad y ampliada a las ITS.

Hay una marcada diferencia en los conocimientos sobre el VIH/SIDA por sexo y grupo etario. Concordando con Puig y Montalvo ${ }^{(20)}$, las mujeres tienen menos conocimiento sobre el tema que los varones, y con ello, tal como lo señalan Ponce et al. ${ }^{(6)}$, se convierten, peligrosamente, en un grupo con más vulnerabilidad al VIH. Por el contrario, cabe señalar que estos resultados difieren de lo señalado por Ponce y Nuñez ${ }^{(22)}$ en un estudio con pueblos indígenas de México sobre VIH/SIDA, quienes destacan que el nivel de conocimiento es menor entre los varones como consecuencia de factores culturales e institucionales. Por su parte, el acceso a la educación que tienen los adolescentes Shuar permite que dispongan de mayor información sobre el VIH/SIDA, pero no se evidenció mayor contacto con otras culturas, vínculo que, en opinión de Puig y Montalvo ${ }^{(20)}$, aporta mayor conocimiento. Con todo, las limitaciones para generar y administrar la información de salud se deben a las inequidades y obstáculos en el acceso al derecho a la salud ${ }^{(13)}$.

Tal como lo encontrado por Puig y Montalvo ${ }^{(20)}$, existe duda y confusión entre VIH/SIDA. En algunos casos, ambos términos son identificados como enfermedad y unos pocos responden de forma precisa; sin embargo, todos reflejan su percepción negativa (terrible, incurable y mortal) como en el estudio de Castro et al. ${ }^{(15)}$ con una comunidad Wayuu en Colombia, en donde los participantes de su estudio hicieron alusión al VIH y al SIDA como sinónimos y como enfermedades malas, incurables y que matan.

El conocimiento sobre transmisión del VIH/ SIDA es limitado, el contagio se asocia a las relaciones sexuales sin protección, al paso de la enfermedad de la madre a los hijos durante el embarazo o por compartir agujas contaminadas. Además, mencionan a los sujetos de transmisión: personas infectadas, portadoras o enfermas. Esta información concuerda con lo señalado en otros estudios $^{(5,20,23)}$ realizados con indígenas de Bolivia, Ecuador y Colombia, en donde los participantes han asociado el contagio a la falta de protección en las relaciones sexuales, y que el paso de la enfermedad de la madre a hijo sería un factor de riesgo importante.

Al igual que otros estudios ${ }^{(5,21)}$ se observan concepciones erróneas sobre la transmisión, catalogados a veces como mágico-religiosos ${ }^{(23)}$, tales como: contagiarse por estrechar la mano de un infectado, compartir el asiento, uso común de platos, cubiertos y sanitarios, por picadura de un zancudo, por compartir comida, por el uso de baños públicos o al contacto de un beso. Practicar la masturbación o la falta de aseo vinculada a relaciones sexuales como fuente de transmisión fueron creencias no mencionadas en la literatura y la presencia de "creencias no documentadas sobre la transmisión del VIH"(24), denotan el nivel de instrucción y la influencia de los mitos y factores culturales propios de cada grupo étnico.

Tal comootrasexperiencias ${ }^{(20)}$, enlosinformantes, el conocimiento sobre signos y síntomas es parcial, y entre otros se menciona: variaciones en la pérdida de peso, el apetito, diarrea, manchas en la piel. De igual forma, las limitaciones de conocimientos y la presencia de tabúes ${ }^{(15,16)}$ se constituyen en factores peligrosos por la consecuente proliferación del virus por conductas riesgosas ${ }^{(17)}$.

Como en toda investigación cualitativa, la sola presencia de los investigadores puede influir en las respuestas de los participantes, las investigadoras aseguran que durante todo el proceso se ha tenido el cuidado de no incurrir en ese error y evitar sesgos. 
Fue una limitación para este estudio la escasa literatura científica actualizada sobre VIH/SIDA y etnia, por lo que los resultados de la presente investigación deben ser transferibles a poblaciones más amplias, a la vez que considerar las condiciones bajo las cuales se recolectaron los datos y aplicar similares características de los participantes ${ }^{(25)}$.

\section{CONCLUSIONES}

La comunidad Shuar de Morona Santiago conoce que el VIH y el SIDA avanzan en forma peligrosa, sin embargo, la información y el conocimiento sobre ello es limitado, circula como rumor, a pesar de que la información técnica proviene de la escuela, de los medios de comunicación tradicionales y de los centros de salud entregada por los Técnicos de

\section{REFERENCIAS}

1. ONUSIDA [Internet]. Ginebra: Programa Conjunto de las Naciones Unidas sobre el VIH/ SIDA. Poner fin a la epidemia de sida para 2030; c2019 [citado 9 feb 2018]. Disponible en: http:// www.unaids.org/es/Homepage

2. OMS [Internet]. Ginebra: Organización Mundial de la Salud. 10 datos sobre el VIH/Sida; c2017 [citado 9 feb 2018]. Disponible en: http://www. who.int/features/factfiles/hiv/es/

3. MINSA, UNFPA. Una aproximación cualitativa a la prevención del VIH/SIDA en dos comunidades nativas de Ucayali [Internet]. Lima: Ministerio de Salud, Fondo de Población de las Naciones Unidas; 2009 [citado 9 feb 2018]. 57 p. Disponible en: http://bvs.minsa.gob.pe/local/MINSA/1034_ MINSA1430.pdf

4. Vinaccia S, Quiceno JM, Gaviria AM, Soto AM, Gil MD, Ballester R. Conductas Sexuales de Riesgo para la Infección por Vih/Sida en Adolescentes Colombianos. Ter Psicol [Internet]. 2007 Jun [citado 5 feb 2018]; 25(1): 39-50. Disponible en: http://dx.doi.org/10.4067/S0718480820070001000030

5. Zambrano R, Castro D, Lozano M, Gómez N, Rojas C. Conocimientos sobre VIH y comportamientos en Salud Sexual y Reproductiva en una comunidad indígena de Antioquia. Investig andina [Internet].
Atención Primaria de Salud (TAPS) que visitan las localidades.

Se interpreta la enfermedad desde los propios saberes y creencias. Los hombres y los adolescentes conocen mejor el virus y la enfermedad; es claro el conocimiento, aunque reducido a las manifestaciones clínicas; conocen que el virus ataca la inmunidad, que la enfermedad es muy contagiosa, sin embargo, persisten algunos mitos sobre el contagio.

Cuando la oportunidad se da la gente expresa su interés y la disposición a conocer mejor el VIH/ SIDA, que la perciben como una enfermedad terrible, incurable, mortal y muy fácil de contagiar, en cuyo caso, sin conocer la ruta a seguir.

Se perciben mitos en la comunidad de que las personas diagnosticadas con el virus tienen un deseo sexual exacerbado.

2013 [citado 5 feb 2018]; 15(26): 640-52. Disponible en: http://www.redalyc.org/articulo. oa?id=239026287001

6. Ponce P, Muñoz R, Stival M. Pueblos indígenas, VIH y políticas públicas en Latinoamérica: una exploración en el panorama actual de la prevalencia epidemiológica, la prevención, la atención y el seguimiento oportuno. Salud Colect [Internet]. 2017 sep [citado 6 nov 2018]; 13:537-54. Disponible en: https://www.scielosp.org/article/ scol/2017.v13n3/537-554/es/

7. ONUSIDA. Estado del Arte sobre VIH y Pueblos Indígenas en América Latina [Internet]. Ginebra: Programa Conjunto de las Naciones Unidas sobre el VIH/SIDA; 2016 [citado 22 may 2019]. 53 p. Disponible en: http://onusidalac.org/1/ images/Resumen-Ejecutivo-FINAL-ONUSIDASIPIA-30-04-2016.pdf

8. PAHO [Internet]. Washington D.C: Organización PanamericanadelaSalud. Salud en las Américas 2012 - Determinantes e inequidades en salud. [citado $9 \mathrm{feb}$ 2018]. Disponible en: http://www.paho.org/saluden-las-americas-2012/index.php?option=com_co ntent\&view=article\&id=58:health-determinantsand-inequalities\&Itemid $=165 \&$ lang=es

9. Betancourt F, Pinilla P. Apreciaciones sobre el contexto sociocultural del VIH-Sida en las comunidades indígenas en Colombia. Desacatos [Internet]. 2011 abr [citado 9 feb 2018]; (35): 75-86. Disponible en: http://www.scielo.org.mx/ 
scielo.php?script=sci_abstract\&pid=S1607-050X2 011000100006\&lng=es\&nrm=iso\&tlng=es

10. OMS [Internet]. Ginebra: Organización Mundial de la Salud. Capítulo 3: VIH-SIDA resistir a un agente mortífero; c2019 [citado 9 feb 2018]. Disponible en: http://www.who.int/whr/2003/ chapter3/es/

11. MSP [Internet]. Quito: Ministerio de Salud Pública. Tu bebé sin VIH: Hazte la prueba; c2017 [citado 9 feb 2018]. Disponible en: http://www. salud.gob.ec/este-viernes-01-de-diciembre-sepresentara-la-campana-tu-bebe-sin-vih-hazte-laprueba-2/

12. ONUSIDA [Internet]. Ginebra: Programa Conjunto de las Naciones Unidas sobre el VIH/ SIDA. Estimaciones de VIH y SIDA; c2019 [citado 5 feb 2018]. Disponible en: http://www. unaids.org/es/regionscountries/countries/ecuador

13. Alderete E. Salud y pueblos indígenas. Quito: Ediciones Abya-Yala; 2004. 166p.

14. Betancourt Y, Díaz Z, Castañeda I. Una mirada género sensible a la transmisión del virus de la inmunodeficiencia humana en poblaciones clave. Rev Cuba Salud Pública [Internet]. 2018 Jul-Sept [citado 6 nov 2018]; 44(3): e1180. Disponible en: https://www.scielosp.org/article/rcsp/2018.v44n3/ e1180/

15. Castro D, Gamella J, Gómez N, Rojas C. Caracterización de la Situación del VIH/SIDA en los Wayuu de Colombia: Una aproximación a sus percepciones. Chungará Arica [Internet]. 2017 mar [citado 9 feb 2018]; 49(1): 1-11. Disponible en: https://scielo.conicyt.cl/pdf/ chungara/2017nahead/aop0117.pdf

16. Campojó N. Nivel de conocimiento sobre atención a pacientes con VIH/SIDA en estudiantes y docentes de la Escuela de Estomatología, Universidad Nacional Toribio Rodríguez de Mendoza de Amazonas, Chachapoyas-2018 [Tesis]. Perú: Universidad Nacional Toribio Rodríguez de Mendoza de Amazonas, Chachapoyas; 2018. 69 p. Disponible en: http://repositorio.untrm.edu.pe/ handle/UNTRM/1455

17. Calvillo C, Rodríguez A. Conocimientos y comportamientos en salud sexual de estudiantes indígenas guatemaltecos residentes en una comunidad rural. Rev Cuba Med Gen Integral [Internet]. 2014
Oct-Dic [citado 9 feb 2018]; 30(4): 467-480. Disponible en: http://scielo.sld.cu/scielo.php?script=sci_ arttext\&pid=S0864-21252014000400008

18. GAD, Sevilla Don Bosco. Plan de Desarrollo y Ordenamiento Territorial [Internet]. Sevilla: Parroquial Sevilla Don Bosco; 2019 [citado 9 feb 2018]. 190 p. Disponible en: http://app.sni.gob. ec/sni-link/sni/PORTAL_SNI/data_sigad_plus/ sigadplusdocumentofinal/1460016370001_ P D O T \% 20 S E V I L L A \% 20 D O N \% 20 BOSCO\%202014\%202019_30-10-2015_22-1306.pdf

19. Hernández R, Fernández C, Baptista P. Metodología de la investigación. 5th ed. México: McGraw-Hill; 2010. 656 p.

20. Puig C, Montalvo P. Infecciones de transmisión sexual, VIH y Sida: una aproximación a conocimientos, actitudes y prácticas de poblaciones adultas y adolescentes indígenas en las tierras bajas de Bolivia. Desacatos. 2011; (35): 41-58.

21. FCI, ECUARUNARI, FLACSO. En la Intimidad del Buen Vivir: Una aproximación a conocimientos, actitudes y prácticas sobre ITS, VIH y SIDA de los pueblos indígenas del Ecuador [Internet]. Quito: FCI Ecuador; 2009 [citado 9 feb 2018]. 100 p. Disponible en: https://www.msh.org/sites/msh. org/files/intimidad_del_buen_vivir.pdf

22. Ponce P, Núñez G. Pueblos indígenas y VIH-Sida. Desacatos [Internet]. 2011 ene-abr [citado 5 feb 2018]; (35): 7-10. Disponible en: http://www. redalyc.org/articulo.oa?id=13919375001

23. Ortiz J, Argudo M, Palacios E, Vega B, Jiménez D, Campoverde $\mathrm{M}$ et al. Percepciones sobre la salud sexual y reproductiva de las mujeres indígenas Kichwas y Shuaras. Ecuador, 2016. Rev Fac Ciencias Méd Univ Cuenca. 2016; 34(2): 21-31.

24. ONUSIDA. El SIDA en cifras 2015 [Internet]. Ginebra: Programa Conjunto de las Naciones Unidas sobre el VIH/SIDA; 2015 [citado 5 feb 2018]. 12 p. Disponible en: http://www.unaids. org/sites/default/files/media_asset/AIDS_by_the_ numbers_2015_es.pdf

25. Castillo E, Vásquez M. El rigor metodológico en la investigación cualitativa. Colom Med [Internet]. 2003 [citado 20 abr 2019]; 34(39)3: 164-167. Disponible en: http://www.redalyc.org/ pdf/283/28334309.pdf 